\title{
Frequency Response from Solar PV: A Dynamic Equivalence Closed-Loop System Identification Approach
}

\author{
Mehdi Ghazavi Dozein, Gilles Chaspierre, Student Members, IEEE, Pierluigi Mancarella, Senior \\ Member, IEEE, Patrick Panciatici, Thierry Van Cutsem, Fellow, IEEE
}

\begin{abstract}
Analysis of the frequency response of integrated transmission-distribution networks with deep penetration of solar photovoltaic (PV) generation faces major challenges due to the complexity emerging from dynamic models of the numerous and diverse PV units involved. This work proposes converterbased dynamic equivalent models for both distributed (distribution network-connected) and large-scale (transmission network-connected) PV units which take into account practical issues such as measurement and coordination delays. Differently from previous work that adopted open-loop identification, the unknown model parameters are identified here through a novel closed-loop identification process based on least-square minimization. This allows capturing the continuous interaction between system and PV responses, thus improving the outcome of the overall frequency response model. The proposed models are validated with real data from the August 2018 separation event in Australia. The results demonstrate the excellent performance of the proposed models in determining the frequency response from $P V$ in both transmission and distribution networks, hence paving the way to its adoption in frequency stability analysis in lowcarbon grids dominated by frequency-responsive renewables.
\end{abstract}

Index Terms-- Cascading failures, Dynamic equivalencing, Frequency stability analysis, PV frequency response, System identification, Distributed energy resources.

\section{INTRODUCTION}

$\mathrm{T}$ HE increasing penetration of renewable energy sources (RES) with power-electronics interfaces and the associated reduction of conventional synchronous generators (SGs) increase the risk of system frequency instability due to reduction of system inertia and primary frequency response resources [1-3]. Low-inertia conditions in RES-rich power systems may in fact result in a high rate of change of frequency (RoCoF) following disturbances which may rapidly take the system frequency outside the emergency frequency band [4-5]. Moreover, in systems with great penetration of distributed and/or large-scale photovoltaic (PV) units, secondary PV tripping (SPVT) due to under-frequency

This work was partially supported by AusNet Services and the Melbourne Energy Institute. M. Ghazavi Dozein and P. Mancarella are with School of Electrical and Electronic Eng., The University of Melbourne, Australia. (E: mghazavi@student.unimelb.edu.au; pierluigi.mancarella@unimelb.edu.au). G. Chaspierre is with Dept. of Elec. Eng. and Comp. Science, University of Liège, Belgium (E: g.chaspierre@uliege.be). P. Panciatici is with Research \& Development Department of RTE, France (E: Patrick.panciatici@rtefrance.com), and T. Van Cutsem is with Fund for Scientific Research (FNRS), University of Liège, Belgium (E: T.VanCutsem@uliege.be). protections [4] may also occur. All of these can in turn impinge on system frequency stability even further [4-5]. In a synchronously interconnected system, a frequency disturbance might then also potentially spread across the grid and cause large and fast changes in the flow of an interconnector; this could eventually end up in activation of the interconnector's protection schemes and even system separation [4-6]. Such fast and complex dynamics and frequency-dependent cascading failures have already been experienced in RES-rich systems, e.g., in Great Britain [7]. It is therefore crucial to build suitable aggregated models of frequency-responsive components and frequency-dependent protection schemes to be able to capture RES-rich system dynamics.

Short-term frequency stability analysis is usually performed via either electromagnetic transient (EMT) models or transient stability models, which consist of differential algebraic equations (DAEs) [8]. Given that PV units either at the distribution level (distributed PV units) or the transmission level (PV farms) are required to deliver over-frequency response according to grid-codes [9-10], they effectively actively impact on and contribute to the system frequency dynamics following disturbances. It is then essential to employ suitable dynamic models of PV units to prevent missing their corresponding impact on system frequency characteristics, especially for deep PV penetration levels where their contribution to frequency response may be substantial. However, the high degree of complexity associated with detailed dynamic modelling of a PV array and its energy conversion systems for numerous (and often unknown in number and characteristics) PV units results in major difficulty in terms of performing combined transmissiondistribution frequency stability analysis. This calls for development of suitable dynamic models of PV units which could effectively decrease the complexity of dynamic analysis while capturing PV participation in frequency control.

Several solutions have so far been proposed to handle the complexity of frequency stability analysis imposed by dynamic model of system components, such as dynamic phasors [11], modal schemes [12], frequency-reliant system equivalents using Prony analysis [13], coherency-based methods [14], and simulation-based (measurement-based) algorithms [8]. At the transmission level, previous investigations aimed to increase computational efficiency in different ways, ranging from simplification of SG model [15] 
or dynamic equivalencing of a group of SGs through coherency-based methods [16], to development of dynamic equivalent models for active distribution networks in RES-rich systems [17]. In fact, the literature on integrated transmissiondistribution dynamic modelling has mainly dealt with complexity emerging from synchronous-based components of the transmission grid or its passive elements (e.g., transmission lines). As for on dynamic equivalent models of aggregated PV units, previous works have mainly focused on voltage disturbances, with particular focus on ride-through capability, introducing new methods such as equivalent impedance modelling, correlation clustering methods, and deep learning-based methods [18-21]. However, given recent developments in grid codes [9-10], PV units are now also required to deliver frequency response. Previous equivalent models are not able to evaluate the aggregated frequency response from PV units, as they were developed for response to voltage disturbances, with focus on reactive power control. Hence, with fast increasing penetration of PV, there is a clear and pressing need for methods that can deal with the complexity of dynamic modelling of aggregated PV units of different scales and locations in frequency stability analysis.

On the above premises, this paper presents a novel dynamic equivalent model of aggregated large-scale PV farms to decrease the complexity of frequency stability analysis emerging from numerous PV power plants. The proposed dynamic model is an inverter-based model which maintains the generic model of PV plants in an equivalent model. This model is then developed to consider possible technical issues, such as coordination and measurement delays, which might not allow PV plants to effectively participate in frequency control. The proposed model is then further developed to construct a dynamic equivalent model for aggregated distributed PV units too and considering distribution network infrastructure. Several methods can be used to parametrize the proposed models, such as system truncation approach [22], artificial neural network-based schemes [23], or simulationbased (measurement-based) system identification techniques [24]. In the context of dynamic equivalencing, a study zone refers to the system area/components for which one aims to obtain an equivalent model or lower-order model (e.g., PV units in this work) [8], [25]. With respect to simulation-based identification methods, most previous research has employed an open-loop identification algorithm in which the measured frequency is a pre-defined, invariant input signal. This means that the continuous impact of the studied zone on the system frequency dynamics is effectively ignored. In the case of massive PV penetration in transmission and distribution networks, however, frequency response provision from PV might have a significant impact on system frequency. Therefore, dynamic equivalencing of transmission-connected as well as distributed PV units with open-loop identification can potentially cause inaccuracy in both the equivalent model and the frequency dynamics. Furthermore, this may cause inaccurate analysis of frequency-dependent emergency mechanisms such as under-frequency load shedding (UFLS) and SPVT. A novel closed-loop identification method is then proposed in this paper which employs a simplified system model to reproduce the frequency during the event under study, and then is used as an input signal for least-square optimization problem, thereby capturing the continuous impact of aggregated PV frequency response on the system frequency, and consequently increasing the accuracy of the dynamic equivalent models. The proposed aggregated dynamic equivalent models for distributed and transmissionconnected PV units are evaluated through a comprehensive study on the August 2018 separation event in Australia [26].

The main contributions of the paper are summarized below:

- Increase the accuracy of existing dynamic equivalent models through a novel closed-loop system identification approach based on least-square minimization: this will become more and more crucial with increasing penetration of frequency-responsive PV, as their response might affect significantly the whole system's frequency dynamics.

- Identify the most important parameters which affect the aggregated PV frequency response: thereby reducing the number of unknown parameters in dynamic equivalencing and speeding up the identification process.

- Propose intuitive aggregated dynamic equivalent models for PV units which preserve the underlying PV physical modelling: such intuitive models are an advantage in dynamic equivalencing, as described in [24-25]. In addition, the proposed model can be seamlessly applied to both transmission- and distribution-connected PV.

- Account for technical response delays and their corresponding impacts on the aggregated frequency response from numerous PV units: technical delays in dynamic equivalencing of PV units have not been studied at all in previous works, while they may be essential to capture system-level response, impacts and benefits.

The paper is organized as follows: Section II presents the proposed aggregated dynamic equivalent models for utilityscale and distributed PV. Our novel closed-loop system identification approach is then introduced in Section III, while its performance is evaluated in the case studies of Section IV. Finally, Section V discusses key concluding remarks.

\section{AgGREGATED DYNAMIC EQUIVALENT MODELS FOR UTILITY-SCALE AND DISTRIBUTED PV UNITS}

\section{A. Aggregated dynamic equivalent model for PV farms}

For frequency stability analysis, it is possible to aggregate power generating units of one type, PV units in this work, in one dynamic equivalent model [27]. The proposed converterbased dynamic equivalent model, shown in Fig. 1, captures the aggregated impact of frequency responses from transmissionconnected PV farms on system frequency characteristic. This aggregated dynamic equivalent model is intuitive as it keeps the generic model of transmission-connected PV unit which leads to enhanced flexibility in PV frequency control [28-29]. The proposed model consists of four different parts: 1) aggregated PV array averaged model, 2) current unidirectional DC-DC converter, 3) DC-link capacitor, 4) three-phase gridside DC-AC converter (GSC). 


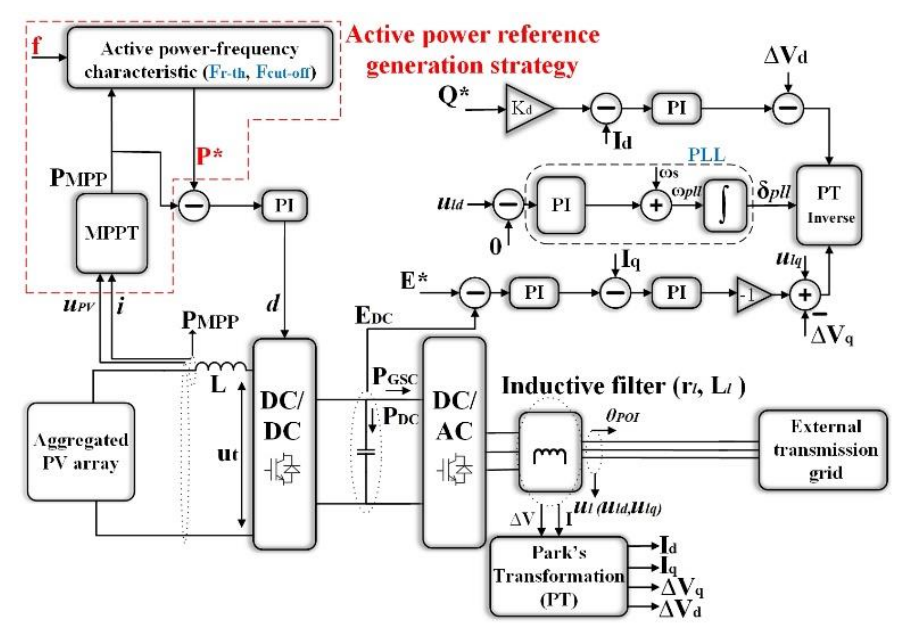

Fig. 1. Proposed aggregated dynamic equivalent model for PV farms.

The GSC is responsible to control the DC-link voltage at the rated value $\left(E^{*}\right)$, which is highly correlated with reactive power output control. The PV-side DC-DC converter manages the active power output and therefore possible frequency response. With regards to converter-level control loops, several proportional integral $(P I)$ controllers are used to control $d$ - $q$ components of output current $\left(I_{d}, I_{q}\right)$, and DC-link voltage $\left(E_{D C}\right)$. The proposed model synchronizes with the external grid via phase-locked loop (PLL). Ideally, the estimated angle $\left(\delta_{P L L}\right)$ and radial frequency $\left(\omega_{P L L}\right)$ by PLL should be equal to the terminal voltage angle $\left(\theta_{P O I}\right)$ and system radial frequency $\left(\omega_{s}\right)$, respectively (i.e., $\delta_{P L L} \approx \theta_{P O I}, \omega_{P L L} \approx \omega_{s}$ ).

A well-known PV "averaged" model, so-called Norton equivalent model, is used in this work to model PV array dynamic behaviour. The dynamic equations of the current flowing through PV-side converter are as follows:

$$
\begin{gathered}
L \frac{d i}{d t}+u_{t}=u_{P V} \\
u_{t}=d E_{D C}
\end{gathered}
$$

where $d$ is the duty cycle of DC-DC converter. Assuming negligible switching power loss, the power flowing through DC-AC converter $\left(P_{G S C}\right)$ can be computed as below:

$$
P_{G S C}=P_{P V}-P_{D C}
$$

where

$$
P_{D C}=\frac{1}{2} C \frac{d}{d t} E_{D C}^{2}
$$

where $P_{D C}$ is the consumed power by the DC-link capacitor (C). Finally, the voltage and current differential equations of GSC can be expressed in $d-q$ coordinates as below:

$$
\begin{aligned}
& u_{c l q}-u_{l q}=r_{l} I_{q}+L_{l} \frac{d I_{q}}{d t}+\omega_{s} L_{l} I_{d} \\
& u_{c l d}-u_{l d}=r_{l} I_{d}+L_{l} \frac{d I_{d}}{d t}-\omega_{s} L_{l} I_{q}
\end{aligned}
$$

where $\left(r_{l}, L_{l}\right)$ characterizes the filter at the grid-side converter terminal, $\left(u_{l d}, u_{l q}\right)$ are the $d$-axis and $q$-axis components of the grid voltage, $\left(u_{c l d}, u_{c l q}\right)$ are the $d$-axis and $q$-axis components of the converter voltage at AC-side. Finally, the PV plant model shown in Fig. 1 consists of 14 state variables (without considering technical delays) - see also [30].

\section{B. Aggregated dynamic equivalent model for distributed PVs}

It is important to take into account distribution lines and transformers in dynamic equivalencing of distributed PV units. To this end, the aggregated dynamic equivalent model of distributed PV also includes, additionally to the model illustrated in Fig. 1 (valid for transmission-connected PV), an equivalent impedance model $\left(R_{D}, X_{D}\right)$ of distribution lines and transformers connected in series between the terminal inductive filter and the external grid. The reader can refer to [31] for further details on control loops based on (1)-(6) for energy conversion systems used in the proposed models.

\section{Unknown parameters of the proposed equivalent models}

PV units, either at distribution or transmission levels, can commonly respond to over-frequency conditions according to their active power-frequency characteristic as shown in Fig. 2. A PV unit works typically at its maximum power point $\left(P_{M P P}\right)$ when the frequency is within the normal operating band. A PV unit delivers over-frequency response through its output reduction once the system frequency is above frequency response threshold $\left(F_{r-t h}\right)$. The PV unit then participates into over-frequency control with full capacity if the frequency is beyond the cut-off frequency (i.e., $f \geq F_{\text {cut-off }}$ ).

It is worth noting that the parameters of active powerfrequency curve for PV units depend on their locations (e.g., transmission level or distribution level) and grid-code requirements and may differ from one another. At transmission level, the frequency response provided from different PV farms may differ from each other mainly for two reasons. First, grid-codes determine the parameters of active power-frequency curve (i.e., $F_{r-t h}, F_{c u t-o f f}$ ) for large-scale PV farms. Thus, grid-scale PV units installed in different years might have different setting, as grid-codes are usually subject to change to ensure that system remains robust and reliable over years. Considering the grid-code requirements in Australia [9], for example, PV units installed prior to 2015 are not required to provide over-frequency control at all, while those installed after 2016 are required to deliver overfrequency response in the case of contingencies. Secondly, the frequency deviation sensed by grid-scale PV units located at different points differs from each other. This difference mainly emerges from uneven inertia distribution in the system, various RoCoF values at different locations following contingencies, and the difference in electrical impedance between fault point and PV locations. This can potentially lead to differences in response provision from PV units connected to different locations. Therefore, the aggregated frequency response from dynamic equivalent model is different from the frequency response provided by each individual PV plant.

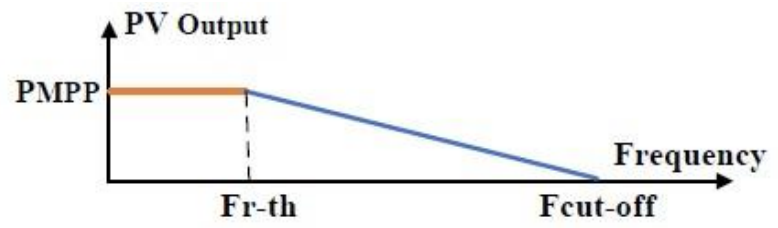

Fig. 2. Typical active power-frequency curve for PV units [10]. 
The active power-frequency characteristic of the proposed dynamic equivalent model (i.e., $F_{r-t h}, F_{c u t-o f f}$ ), as well as its aggregated PLL delay $\left(\delta_{P L L}\right)$, thus need to be adjusted to capture the aggregated frequency response from multiple PV plants. Similarly, distributed PV units are also connected to different locations and they usually follow different frequency requirements since they have been installed in different years. So, it is also required to find the unknown parameters in the proposed dynamic equivalent model of distributed PVs (i.e., $\left.F_{r-t h}, F_{c u t-o f f}, \delta_{P L L}, R_{D}, X_{D}\right)$. Finally, other parameters in equations (1)-(6) have been adjusted so as to guarantee the stability of converter-level control loops.

\section{Technical issues in frequency response provision from utility-scale PV farms}

Transmission-connected PV power plants are usually equipped with power plant controllers to regulate their active and reactive power outputs so that they behave like a single large generation unit [10]. Indeed, the power plant controller is aimed to coordinate individual inverters to provide typical large power plant features, for example primary frequency response in this work. From a practical point of view, transmission-connected PV units might not be able to participate in primary frequency response due to technical issues such as coordination and measurement delays [26]. This is a potential reason for differences in frequency responses provided by various grid-scale PV units. Such practical issues must be considered in the dynamic equivalent model of utilityscale PV farms to avoid impacting on model accuracy. In this work, the active power reference generation strategy, shown in Fig. 1, is then developed further to take into account possible measurement and coordination delays. Fig. 3 shows how measurement and coordination delays are modelled when generating the reference signal through two generic exponential delay functions in the Laplace domain with time delays $\left(r_{m}, r_{c}\right)$, respectively. Finally, other parameters in equations (1)-(6) have been adjusted so as to guarantee the stability of converter-level control loops.

\section{Closed-LOOP System IDENTIFICATION PROCESS OF THE

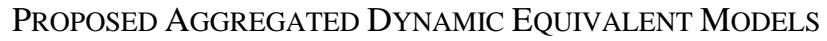

The unknown parameters of the proposed dynamic equivalent models are grouped in a vector $\boldsymbol{\theta}$ (namely $\boldsymbol{\theta}=$ $\left[F_{r-t h}, F_{c u t-o f f}, \delta_{P L L}\right]$ for aggregated large-scale PV farms and $\boldsymbol{\theta}=\left[F_{r-t h}, F_{\text {cut-off }}, \delta_{P L L}, R_{D}, X_{D}\right]$ for distributed PV units) and adjusted in the least-square sense so as to keep the simulated frequency response from aggregated PV units as close as possible to the frequency response observed during the event under study. Regarding the dynamic equivalencing of large-scale PV farms, the $\boldsymbol{\theta}$ vector should be modified to $\left[F_{r-t h}, F_{c u t-o f f}, \delta_{P L L}, r_{m}, r_{c}\right]$ to contain the unknown time delays as well. It is also suggested to merge measurement and coordination delays together since the aggregated time delay affects the performance of PV units in frequency response provision, thereby speeding up the least-square minimization process.

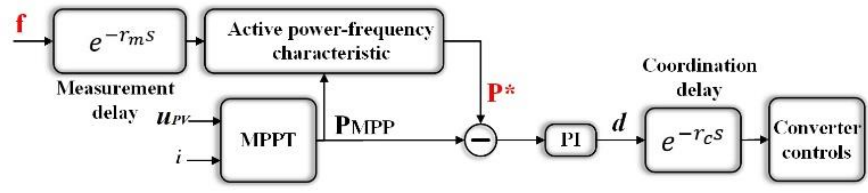

Fig. 3. Proposed active power reference generation strategy including measurement and coordination delays.

In this work, the following objective function is considered:

$$
\varepsilon_{P}(\boldsymbol{\theta})=\frac{1}{N} \sum_{k=0}^{N}[P(k)-\hat{P}(k, \boldsymbol{\theta})]^{2}
$$

under the following constraint:

$$
\boldsymbol{\theta}^{L} \leq \boldsymbol{\theta} \leq \boldsymbol{\theta}^{U}
$$

where $P(k)$ is the discrete-time evolution of the aggregated output of PV units during the event under study while $\widehat{P}(k, \boldsymbol{\theta})$ is the simulated discrete-time evolution of the aggregated output of PV units from the proposed dynamic equivalent model, either at transmission level or distribution level. Also, $k$ is the discrete time of simulation solver, $N$ represents the maximum number of discrete times, $\boldsymbol{\theta}^{L}$ is the minimum limit of $\boldsymbol{\theta}$, while $\boldsymbol{\theta}^{U}$ is its maximum limit.

\section{A. The optimization method}

It is difficult to derive an analytical expression of the first derivative of the objective function with respect to $\boldsymbol{\theta}$ since $\hat{P}(k, \boldsymbol{\theta})$ is obtained from time-domain simulation and steered by discontinuous controls. Therefore, a derivative-free, metaheuristic optimization algorithm [32] has been preferred over classic mathematical programming methods. This work employs the differential evolution (DE) algorithm [33]. This is an evolutionary algorithm and population-based optimizer that starts the minimization process by sampling at multiple, randomly chosen initial points. Then, DE generates a new point through a linear combination of three randomly-selected population points. In this work, the local-to-best strategy is used to generate mutant vector $v_{i, g}$ by randomly choosing two population members $x_{r 1, g}$ and $x_{r 2, g}$ as follows:

$$
v_{i, g}=x_{i, g}+F\left(\text { best }_{g}-x_{i, g}\right)+F\left(x_{r 1, g}-x_{r 2, g}\right)
$$

where $x_{i, g}$ is the $i^{\text {th }}$ member of previous population, best $t_{g}$ is the best member of previous population, and $F$ is the mutant constant. If the mutant parameter vector $v_{i, g}$ results in better objective function compared to $x_{i, g}$, then $v_{i, g}$ substitutes for $x_{i, g}$ in the population. Furthermore, at each new generation, the mutation factor $(F)$ is randomly selected in the range of $[0.5,1]$ to improve the DE convergence significantly [27-28]. The population size is a trade-off between probability of convergence and convergence speed. It is advised in [32-33] to select a population size 10 times larger than the number of unknown parameters. The reader can refer to [32-33] for more information on DE algorithm.

\section{B. Proposed closed-loop identification process}

Considering high PV penetration at both transmission and distribution levels, the PV aggregated frequency response and its associated impact on system frequency dynamics may be 
substantial. In fact, as far as PV units are providing frequency response to the system by altering their active power operating point, they are continuously affecting the system frequency dynamics, and this continuous impact is considerable with high PV penetration. Therefore, it is necessary to consider the aforesaid continuous impact while running the identification process to increase the accuracy of dynamic equivalencing. Although the variation of the model parameters throughout an open-loop simulation-based identification process [34] changes the equivalent model active power output, it has no impact on the system frequency signal used for the identification method since it is a pre-defined, invariant input. Therefore, this work introduces a novel closed-loop system identification method, sketched in Fig. 4, to take into account the continuous impact of the aggregated frequency support from PV. As shown, the system frequency during the event under study is reproduced using the angular speed of the equivalent synchronous generator, thereby the input frequency signal of the identification process is no longer a pre-defined, invariant signal. Fig. 4 shows that the study zone (facing overfrequency condition) is constructed by an equivalent SG as well as an equivalent load accounting for the total inertia of the study zone at the pre-contingency time. The rest of the system is modelled as Thevenin equivalent plus an equivalent load. Also, the transmission lines and transformers in the study zone are modelled through an equivalent impedance $(R, X)$. Finally, load sensitivity to frequency is modelled by [35]:

$$
P=P_{0}\left(1+D_{p} \times \frac{\Delta f}{f_{N}}\right)
$$

where

$$
\Delta f=f-f_{N}, D_{p}=\frac{\frac{\Delta P}{P_{0}}}{\frac{\Delta f}{f_{N}}}
$$

where $D_{p}$ is the load damping factor, $P_{0}$ features the base load, $f$ is the measured frequency, $\Delta P$ is the load change associated with frequency variation $\Delta f$, and $f_{N}$ is the system nominal frequency. The frequency of study zone is calculated using the radial speed of the equivalent synchronous machine.

The following points feature in the proposed closed-loop system identification process:

- The input is the simulated frequency deviation $\Delta f$, as from (11), which is a function of the angular speed of the equivalent synchronous generator in the study zone;

- The output is the simulated active power response of the aggregated PV model $\hat{P}(k, \boldsymbol{\theta})$ which will be compared with the actual response in a least-square manner;

- The regressor is the embedded test system (Fig. 4) equations which takes into account the proposed dynamic equivalent models including their unknown parameters.

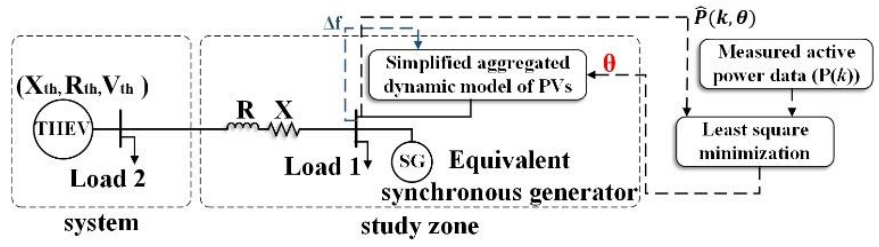

Fig. 4. Proposed closed-loop identification process and associated equivalent systems.
C. Coupling between optimization algorithm and timesimulation tools

Similar to all population-based algorithms, DE generates a large amount of points in the search space. At each new generation of $\boldsymbol{\theta}$, a time-domain simulation has to be run to generate the associated active power output $\hat{P}(k, \boldsymbol{\theta})$. Therefore, the time required to complete a time-domain simulation has a huge impact on the identification processing time, irrespectively of the optimization algorithm selected. To overcome this problem, two options can be considered: 1) a software which requires less processing time to complete a time-domain simulation of the simplified test-system shown in Fig. 4, and/or 2) a simplified dynamic model of PV units should be used which takes into account their interaction with the external grid in response to frequency excursions. Once the unknown parameters are identified, they can be used to fully construct the dynamic equivalent models. These are then finally integrated into a power system simulation tool to reproduce the frequency response from PV farms, as well as distributed PV units, during specific events under study.

\section{CAse Studies}

This section aims to assess the performance of the proposed dynamic equivalent models through a comprehensive study on frequency response provided by PV farms and distributed PV in Queensland (QLD) and South Australia (SA) during the 25 August 2018 separation event in the Australian National Electricity Market (NEM) grid [26].

\section{A. The Australian grid and the August 2018 separation event}

The Australian states and the NEM grid interconnectors are schematically shown in Fig. 5. At the time of the event, multiple strikes on the Queensland-New South Wales Interconnector (QNI) caused a double back flashover to the tower/earth wire, resulting in an unbalanced connection between Queensland (QLD) and New South Wales (NSW). This rapidly led to loss of synchronism between those two states, causing QNI trip and finally QLD separation. Subsequently, the QLD region experienced an over-frequency condition with a frequency overshoot around $50.9 \mathrm{~Hz}$ since it was exporting $865 \mathrm{MW}$ power to NSW, while the rest of the system faced an active power deficit as well as a frequency drop. Generation units in South Australia (SA) started to respond to this frequency drop, which resulted in increased active power flow on the Heywood interconnector from SA to Victoria (VIC). This eventually also activated the Heywood interconnector emergency protection schemes, leading to the interconnector's trip and SA separation around 6 seconds after the QNI trip. The SA separation left the mainland (i.e., VIC and NSW) to experience further frequency drop, as SA was exporting power to VIC. Finally, the frequency in the mainland stabilized due to the activation of 977 MW UFLS as well as primary frequency droop response from SGs [26]. 


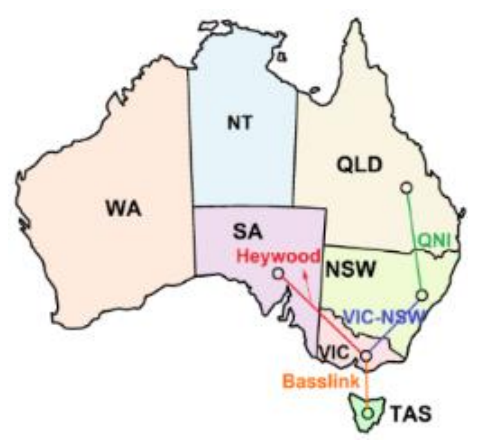

Fig. 5. Australian states and NEM grid interconnectors.

B. Coupling between optimization algorithm and timesimulation tools for the August 2018 separation event

Two simulation tools were used to simulate the aggregated frequency response from PV farms and distributed PV units in QLD and SA, which experienced over-frequency conditions following their separations. Firstly, a simplified equivalent model of the NEM grid, as per Fig. 4, was constructed using the phasor-mode simulator RAMSES [36] for identification purposes. It is worth saying that the proposed equivalencing approach is completely general and scalable to other simulation tools (e.g., [37]). The proposed closed-loop identification process benefits from the RAMSES model since it contains a simplified generic model of inverter-based generators [34], which can be used as a simplified dynamic model of PV farms and distributed PV units throughout the identification process. This work links the proposed identification process to the RAMSES model to update the unknown parameters $(\boldsymbol{\theta})$ of aggregated dynamic equivalent models of both transmission-connected and distributed PV in a least-square minimization procedure. Based on the Australian grid-code [26], PV units must deliver a sustained droop response during over-frequency conditions, meaning that they only respond to frequency overshoot and then they maintain their reduced active power output for 10 minutes while the frequency is recovering. Taking the aforementioned requirement into account, the objective function in (7) is then revised by adding an additional corrective term to make sure that the simulated frequency overshoot is close to the actual frequency overshoot observed during the event in different states (QLD and SA), thereby satisfying the persistent excitation condition in system identification. Therefore, this specific work deploys the following objective function:

$$
\varepsilon_{P f}(\boldsymbol{\theta})=\varepsilon_{P}(\boldsymbol{\theta})+\gamma\left|\Delta f_{\text {smax }}-\Delta f_{\text {amax }}\right|
$$

where $\Delta f_{\text {smax }}$ is the simulated frequency overshoot, $\Delta f_{\text {amax }}$ is the actual frequency overshoot observed during the event in a certain area, and $\gamma$ is a constant coefficient. Once the $\boldsymbol{\theta}$ vector is identified for aggregated dynamic model of both PV farms and distributed PV units, the models are imported into the Australian 14-generator test system [38] in MATLAB/SIMULINK platform, which is a simplified equivalent dynamic model of the Australian NEM grid, so as to reproduce the high-level frequency dynamics observed in the August 2018 separation event. As discussed in [4], [39], the simulated frequency dynamic behaviour in different states well reflects the frequency traces measured during the event.

\section{Frequency control from $P V$ units in Queensland}

At the time of the event, 9 grid-scale PV farms were online in QLD with an active power output of 286.1 MW and total capacity of $586 \mathrm{MW}$. The Australian Energy Market Operator (AEMO) reported that utility-scale PV farms in QLD successfully provided 79 MW aggregated over-frequency response following the QNI trip [23]. The proposed dynamic model is then used to simulate the aggregated frequency response from PV farms in QLD. In fact, the proposed dynamic equivalent model is constructed in lieu of modelling 9 grid-scale PV farms, which improves the computation efficiency. The consideration of 9 grid-scale PV power plants will add at least 126 DAEs to the system dynamic model since every PV unit includes 14 DAEs minimum, while the proposed dynamic equivalent model comprises only 14 DAEs in the case of no delay consideration. The simplified testsystem model is implemented in RAMSES, using data presented in Table I (QLD case), where the study zone is QLD and the rest of the system is the interconnected SA-VIC-NSW system. Table I also presents the parameters deployed in the minimization process. In this work, the optimization process stops when $\varepsilon_{P}(\boldsymbol{\theta})$ reduces less than $0.1 \mathrm{MW}$ during 10 successive $\mathrm{DE}$ iterations. The measurement delay is aggregated to the coordination delay and it is assumed to be zero $\left(r_{m}=0\right)$ so as to accelerate the identification process.

The proposed closed-loop identification process is terminated after 55 iterations in 30 minutes for 35 -second simulated time, using a computer with an Intel(R) i7-6820 HQ quad-core processor @ $2.70 \mathrm{GHz}$, and $16 \mathrm{~GB}$ of RAM. The parameters for equivalent model of PV farms are obtained using the proposed closed-loop identification process and presented in Table II.

TABLE I. PARAMETERS USED FOR DYNAMIC EQUIVALENCING OF DISTRIBUTED AND UTILITY-SCALE PV UNITS

\begin{tabular}{|c|c|c|c|}
\hline Parameter & Symbol & $\begin{array}{c}\text { Value } \\
\text { for QLD } \\
\text { case }\end{array}$ & $\begin{array}{c}\text { Value } \\
\text { for SA } \\
\text { case }\end{array}$ \\
\hline Equivalent resistance [pu] & $R$ & 0.1 & 0.1 \\
\hline Equivalent reactance [pu] & $X$ & 1.089 & 1.089 \\
\hline Equivalent load in QLD [MW] & Load-1 & 5350 & 775 \\
\hline $\begin{array}{c}\text { Equivalent load in SA-VIC-NSW } \\
\text { [MW] }\end{array}$ & Load-2 & 1000 & 250 \\
\hline Load damping factor [\%] & $D_{p}$ & 1 & 4.14 \\
\hline Thevenin equivalent resistance [pu] & $R_{t h}$ & 4.16 & 2.08 \\
\hline Thevenin equivalent reactance [pu] & $X_{t h}$ & 41.16 & 20.8 \\
\hline Thevenin short circuit MVA & $S_{s c}$ & 25000 & 25000 \\
\hline Thevenin equivalent voltage [kV] & $V_{t h}$ & 330 & 330 \\
\hline $\begin{array}{c}\text { Inertial constant of the equivalent } \\
\text { SG [Sec] }\end{array}$ & $H$ & 5 & 5 \\
\hline $\begin{array}{c}\text { Nominal MVA of the equivalent } \\
\text { SG }\end{array}$ & $S_{n o m}$ & 11000 & 4000 \\
\hline $\begin{array}{c}\text { Resistance of the equivalent SG } \\
\text { [pu] }\end{array}$ & $R_{a}$ & 0 & 0 \\
\hline Reactance of the equivalent SG [pu] & $X_{l}$ & 0.15 & 0.15 \\
\hline $\begin{array}{c}\text { Nominal voltage of the equivalent } \\
\text { SG [kV] }\end{array}$ & $V_{t}$ & 330 & 330 \\
\hline Weighted coefficient in (12) & $\gamma$ & 200 & 200 \\
\hline
\end{tabular}


TABLE II. PARAMETERS OF AgGREGATED DYNAMIC EQUIVALENT MODEL FOR PV FARMS IN QLD

\begin{tabular}{|c|c|}
\hline Parameter & Value \\
\hline$F_{r-t h}$ & $50.4895(\mathrm{~Hz})$ \\
\hline$F_{c u t-o f f}$ & $51.44(\mathrm{~Hz})$ \\
\hline$\delta_{P L L}$ & $40 \mathrm{~ms}$ \\
\hline$r_{m}$ & 0 \\
\hline$r_{c}$ & 1.1 seconds \\
\hline
\end{tabular}

Fig. 6 shows the simulated frequency response from aggregated dynamic equivalent model of PV farms in QLD as well as the simulated QLD frequency following the QNI trip. From Fig. 6 it may be seen how the simulated frequency response (71 $\mathrm{MW}$ ) is close to the actual frequency response provided by grid-scale PV units in QLD (79 MW), showing how the proposed dynamic equivalencing approach is able to capture with good fidelity the aggregated frequency response from PV farms during the event. It is worth saying that the over-frequency droop response from aggregated grid-scale PV units is different from typical over-frequency droop response from synchronous generators in Australia. This is because the aggregated PV output does not follow the frequency and it is sustained while the frequency recovers, as from the grid-code requirements, as opposed to the frequency droop response from synchronous generators. Although grid-scale PV units could in principle provide fast frequency response thanks to the high converter controllability, the actual response (79 MW) was delivered slowly due to the coordination delay. Fig. 6 highlights the ability of the proposed model to capture such technical issues, as the simulated frequency (71 MW) response is delivered to the QLD region with a delay of 1.1 seconds.

Figure 7 also shows that grid-scale PV units would have been able to deliver 103.6 MW aggregated frequency response if they had delivered a sustained droop response with no delay. In this case, the QLD frequency overshoot could have been lower than $0.8 \mathrm{~Hz}$ following the QNI trip.

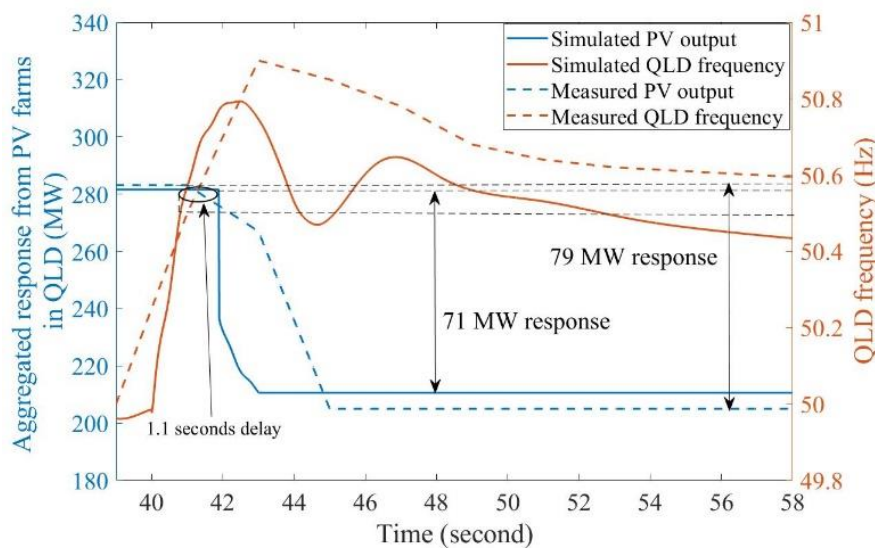

Fig. 6. Frequency response from PV farms and QLD frequency following the QNI trip, for simulated results and actual measured data.

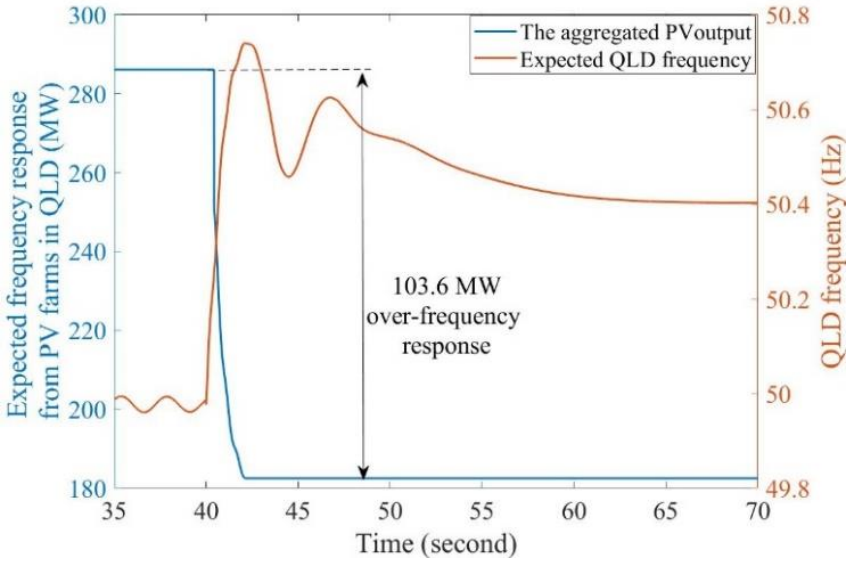

Fig. 7. Expected frequency response from aggregated PV farms in QLD and simulated QLD frequency.

Similarly, the identification process is performed to simulate the aggregated frequency response provided by distributed PV units in QLD. AEMO reported in [26] that the pre-contingency generation by distributed PV was $1043 \mathrm{MW}$ with online installed capacity of $2177 \mathrm{MW}$, while $165 \mathrm{MW}$ over-frequency response from distributed PV was observed in QLD. The vector $\boldsymbol{\theta}$ for dynamic equivalent model of distributed PV in QLD is obtained using the proposed closedloop identification process and presented in Table III (Case-1).

To show the importance of the proposed closed-loop system identification method, as well as the importance of corrective term in (12), we have also identified the unknown parameters for aggregated dynamic equivalent model of distributed PV units in QLD by other identification methods, namely: closed-loop identification approach considering equation (7) as the objective function, where there is no corrective term (Case-2); and the open-loop identification method presented in [34] (Case-3). The parameters identified in Case-2 and Case-3 are also presented in Table III. The QLD distributed PV dynamic equivalent models obtained from the different identification methods have then been integrated into the NEM test system, as explained above.

TABLE III. PARAMETERS FOR AgGREgated DYNAMIC EQUiVALENT MODEL OF DISTRIBUTED PV UNITS FOR VARIOUS CASES

\begin{tabular}{|c|c|c|}
\hline Case & Parameter & Value \\
\hline \multirow{5}{*}{ Case-1 } & $F_{r-t h}$ & $50.521(\mathrm{~Hz})$ \\
\hline & $F_{\text {cut-off }}$ & $51.854(\mathrm{~Hz})$ \\
\hline & $\delta_{P L L}$ & $200 \mathrm{~ms}$ \\
\hline & $R_{D}$ & 0 \\
\hline & $X_{D}$ & 0 \\
\hline \multirow{5}{*}{ Case-2 } & $F_{r-t h}$ & $50.597(\mathrm{~Hz})$ \\
\hline & $F_{c u t-o f f}$ & $52.98(\mathrm{~Hz})$ \\
\hline & $\delta_{P L L}$ & $42.54 \mathrm{~ms}$ \\
\hline & $R_{D}$ & 0 \\
\hline & $X_{D}$ & 0 \\
\hline \multirow{5}{*}{ Case-3 } & $F_{r-t h}$ & $50.48(\mathrm{~Hz})$ \\
\hline & $F_{\text {cut-off }}$ & $53.16(\mathrm{~Hz})$ \\
\hline & $\delta_{P L L}$ & $110.5 \mathrm{~ms}$ \\
\hline & $R_{D}$ & 0 \\
\hline & $X_{D}$ & 0 \\
\hline
\end{tabular}




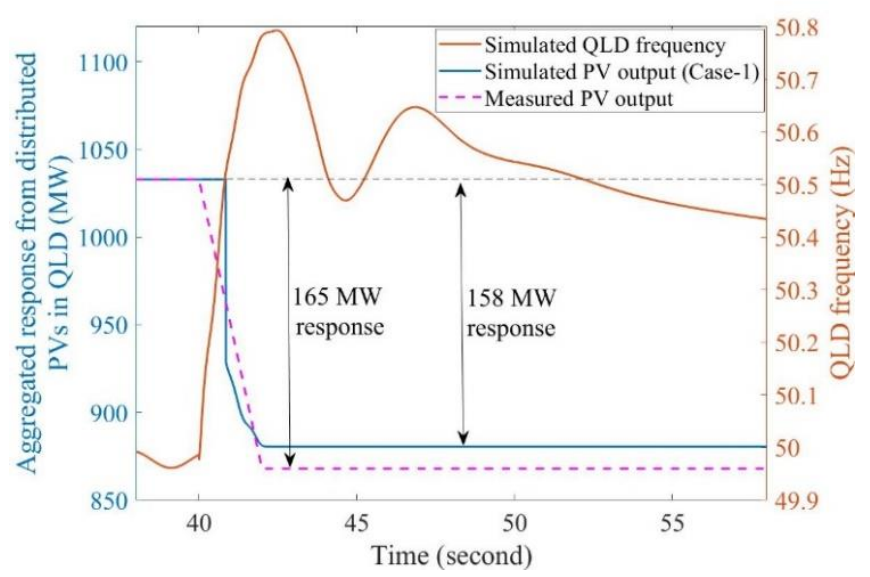

Fig. 8. Aggregated frequency response from distributed PV units in QLD in the simulated August 2018 event (Case-1) and actual measured response.

It should be noted that the identified parameters of the distribution grid equivalent impedance $\left(R_{D}, X_{D}\right)$ are zero in different cases, as grid impedances have no, or very limited, impact on frequency dynamics. Second, Fig. 8 shows around 158 MW simulated frequency response from aggregated distributed PV units in QLD (Case-1), while the actual response was around $165 \mathrm{MW}$. The simulation result validates the proposed dynamic equivalent model in capturing the aggregated dynamic behaviour of distributed PV units.

Third, Fig. 9 depicts the simulated frequency response from aggregated distributed PV units in QLD obtained by different identification methods as defined earlier. As shown, the simulated response obtained via open-loop identification approach (Case-3) is around $133 \mathrm{MW}$, while that of Case-2 is around $105 \mathrm{MW}$. Table IV compares the simulated response in different cases with the measured response observed during the event by using the following error measure:

$$
\text { Error }=\frac{P_{\text {measured }}-P_{\text {measured }}^{k}}{P_{\text {measured }}} \times 100[\%]
$$

where $P_{\text {measured }}$ is the actual response from distributed PVs observed during the event while $P_{\text {measured }}^{k}$ is the simulated response obtained via dynamic equivalent model in Case- $k$ $(k \in\{1,2,3\})$. From Table IV, it can be concluded that the proposed closed-loop system identification approach (Case-1) results in better accuracy in dynamic equivalencing of aggregated distributed PV units. It should be noted that the proposed closed-loop identification process, including equation (12) as its objective function, will be considered in the rest of the simulation case studies to avoid repetition.

TABLE IV. Simulated RESPONSE VS THE ACTUAL RESPONSE

\begin{tabular}{|c|c|c|c|}
\hline Case & $\begin{array}{c}\text { Simulated } \\
\text { response [MW] }\end{array}$ & $\begin{array}{c}\text { Measured } \\
\text { response [MW] }\end{array}$ & Error [\%] \\
\hline Case-1 & 158 & 165 & 4.2 \\
\hline Case-2 & 133.3 & 165 & 19.2 \\
\hline Case-3 & 105.5 & 165 & 36.1 \\
\hline
\end{tabular}

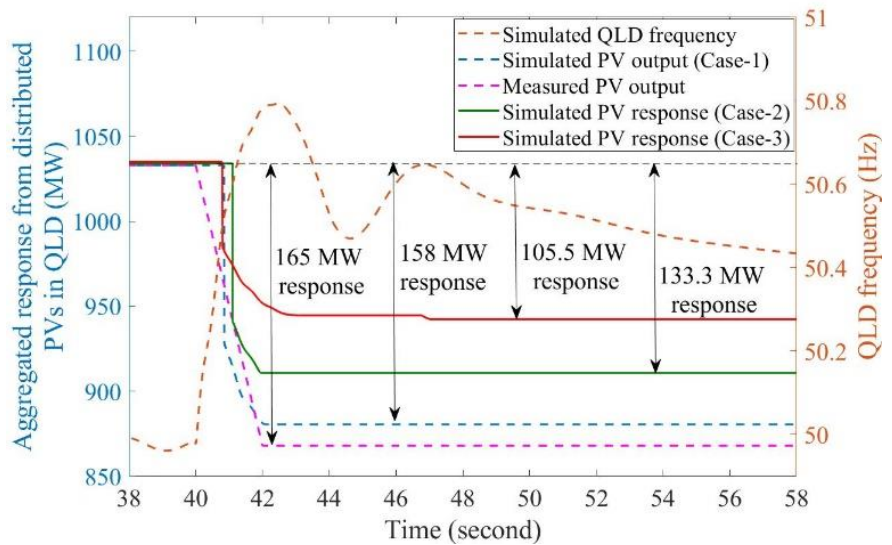

Fig. 9. Aggregated frequency response from distributed PV units in QLD for different cases and actual measured response.

\section{Frequency control from PV in South Australia}

It is reported by AEMO that the single online large-scale PV farm in SA at the time of the event, with active power output of $89 \mathrm{MW}$ and capacity of $110 \mathrm{MW}$, was not able to respond to the frequency overshoot following the Heywood interconnector trip due to a severely large delay around 4 seconds, including measurement and coordination delays [26]. Here we thus aim to understand how much frequency response could have been delivered to the SA system from the gridscale PV farm if there was no technical delay. Fig. 10 depicts how the grid-scale PV unit in SA could have provided $83 \mathrm{MW}$ over-frequency sustained droop response to the SA system, and thus could have resulted in $0.1 \mathrm{~Hz}$ improvement in the SA frequency overshoot after SA separation if there was no delay.

The data in Table I (SA case) is then used for dynamic equivalencing of distributed PV units in SA. The unknown parameters for dynamic equivalent model are obtained by the closed-loop system identification process and presented in Table V. Fig. 11 shows the aggregated frequency response from the proposed dynamic equivalent model as a response to the simulated SA frequency as well as the actual measured PV response: the simulated frequency response (i.e., $64 \mathrm{MW}$ ) is again close to the actual frequency response from distributed PV units in SA (i.e., $60 \mathrm{MW}$ ) reported by AEMO [26].

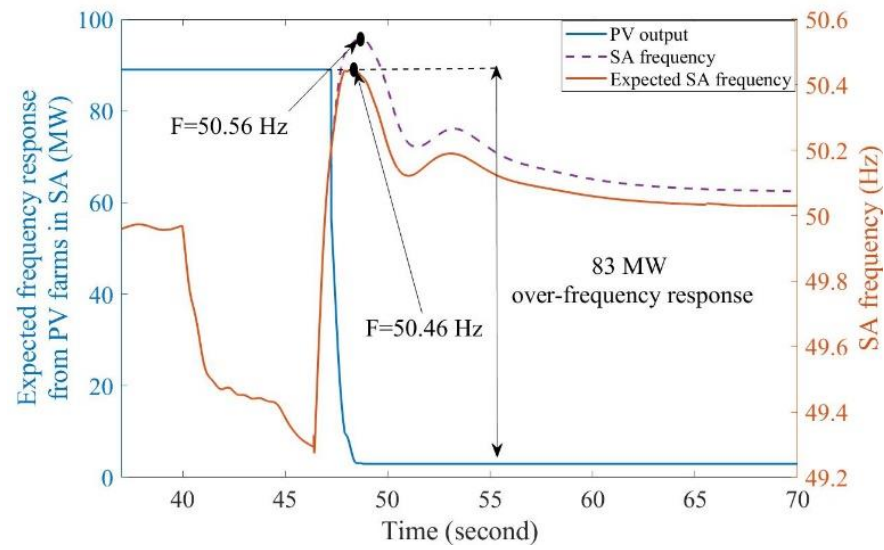

Fig. 10. Expected frequency response from grid-scale PV unit and simulated SA frequency in two different cases. 
TABLE V. PARAMETERS OF AGgREGATED DYNAMIC EQUIVALENT MODEL FOR DISTRIBUTED PV UNITS IN SA

\begin{tabular}{|c|c|}
\hline Parameter & Value \\
\hline$F_{r-t h}$ & $50.28(\mathrm{~Hz})$ \\
\hline$F_{c u t-o f f}$ & $52.4(\mathrm{~Hz})$ \\
\hline$\delta_{P L L}$ & $68 \mathrm{~ms}$ \\
\hline$R_{D}$ & 0 \\
\hline$X_{D}$ & 0 \\
\hline
\end{tabular}

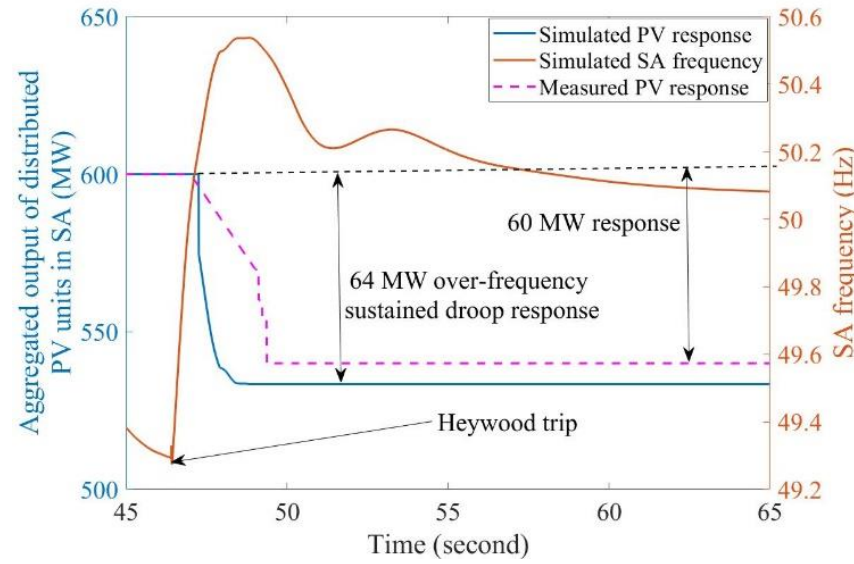

Fig. 11. Simulated/Measured frequency response from distributed PVs in SA following Heywood interconnector's trip and simulated SA frequency.

\section{CONCLUSION}

This paper has proposed novel dynamic equivalent models for transmission-connected and distributed PV units to improve the performance of frequency stability analysis in PV-rich power systems. The proposed models are based on generic converter-based PV dynamic model which retains the physical model of PV units including aggregated PV array model, DC-DC converter, DC-link, DC-AC converter, and their associated control loops. As the aggregated frequency response from several large-scale as well as distributed PV units might differ from what provided by each individual PV unit, the dynamic equivalents have been parametrized through a novel closed-loop system identification process. This employs a simplified equivalent test-system model to simulate the system frequency during the event under study, thus taking into account the continuous impact of PV response on the system frequency and eventually resulting in higher accuracy in model parametrization. The dynamic equivalent model for grid-scale PV farms was then further developed to capture possible technical issues that might deteriorate their frequency response, such as coordination and measurement delays. The results from model validation based on the August 2018 separation event in Australia demonstrate how the proposed approach is able to capture with good fidelity the aggregated frequency response from grid-scale and distributed PV.

The dynamic equivalencing methodology of PV units of different locations and scales we propose here can be deployed by system operators as a powerful tool to accurately evaluate PV aggregated frequency response. This will become increasingly important with larger penetration of distributed and centralized PV plants, thus contributing to reduce the complexity of frequency response analysis and improve system stability and security in low-carbon power systems.

As future work, even though the robustness of the proposed identification approach has been extensively verified by multiple algorithm runs, we aim to test it further against other disturbances and improve its accuracy by considering piecewise active power-frequency characteristics.

\section{REFERENCES}

[1] M. J. Hossain, et al., "Investigation of the Impacts of Large-Scale Wind Power Penetration on the Angle and Voltage Stability of Power Systems," in IEEE Systems Journal, vol. 6, no. 1, pp. 76-84, 2012.

[2] S. Eftekharnejad, et al., "Impact of increased penetration of photovoltaic generation on power systems," in IEEE Trans. on Power Syst., vol. 28, no. 2, pp. 893-901, May 2013.

[3] N. K. Roy and H. R. Pota, "Current Status and Issues of Concern for the Integration of Distributed Generation into Electricity Networks," in IEEE Systems Journal, vol. 9, no. 3, pp. 933-944, 2015.

[4] A. Jalali, M. G. Dozein and P. Mancarella, "Frequency Stability Provision From Battery Energy Storage System Considering Cascading Failure s with Applications to Separation Events in Australia," 2019 IEEE Milan PowerTech, Milan, Italy, pp. 1-6, 2019.

[5] S. Püschel-Løvengreen et al., "Separation event-constrained optimal power flow to enhance resilience in low-inertia power systems," Electric Power Systems Research, vol. 189, 2020.

[6] J. Ma, et al., "Angle Stability Analysis of Power System With Multiple Operating Conditions Considering Cascading Failure," in IEEE Trans. on Power Systems, vol. 32, no. 2, pp. 873-882, March 2017.

[7] National Grid ESO, "Technical report on the events of 9 August 2019", pp. 1-37, September 2019.

[8] A. M. Stankovic and A. T. Saric, "Transient power system analysis with measurement-based grey box and hybrid dynamic equivalents," in IEEE Trans. on Power Systems, vol. 19, no. 1, pp. 455-462, Feb. 2004.

[9] Australian Energy Market Operator (AEMO), "Electricity rule change proposal, generator technical requirement", AEMO Information \& Support Hub, Australia, August 2017.

[10] National Renewable Energy Laboratory (NREL), "Demonstration of essential reliability services by a $300-\mathrm{MW}$ solar photovoltaic power plant”, Technical Report, March 2017.

[11] A. M. Stankovic and T. Aydin, "Analysis of asymmetrical faults in power systems using dynamic phasors," in IEEE Trans. on Power Systems, vol. 15, no. 3, pp. 1062-1068, Aug. 2000.

[12] G. C. Verghese, I. J. Perez-Arriaga and F. C. Schweppe, "Selective Modal Analysis with Applications to Electric Power Systems, Part II: The Dynamic Stability Problem," in IEEE Trans. on Power Apparatus and Systems, vol. PAS-101, no. 9, pp. 3126-3134, Sept. 1982.

[13] Jun-Hee Hong and Jong-Keun Park, "A time-domain approach to transmission network equivalents via Prony analysis for electromagnetic transient analysis," IEEE Trans. on Power Systems, vol. 10, no. 4, pp. 1789-1797, Nov. 1995.

[14] R. Nath, S. S. Lamba and K. S. P. Rao, "Coherency Based System Decomposition into Study and External Areas Using Weak Coupling," in IEEE Trans. on Power Apparatus and Systems, vol. PAS-104, no. 6, pp. 1443-1449, June 1985.

[15] IEEE, "Approved Draft Guide for Synchronous Generator Modeling Practices and Parameter Verification with Applications in Power System Stability Analyses," in IEEE P1110/D07, vol., no., pp.1-101, 2019.

[16] Y. Liang, X. Lin, A. M. Gole and M. Yu, "Improved Coherency-Based Wide-Band Equivalents for Real-Time Digital Simulators," in IEEE Trans. on Power Systems, vol. 26, no. 3, pp. 1410-1417, Aug. 2011.

[17] S. Mat Zali and J. V. Milanović, "Generic Model of Active Distribution Network for Large Power System Stability Studies," in IEEE Trans. on Power Systems, vol. 28, no. 3, pp. 3126-3133, Aug. 2013.

[18] P. Li et al., "High-Precision Dynamic Modeling of Two-Staged Photovoltaic Power Station Clusters," in IEEE Trans. on Power Systems, vol. 34, no. 6, pp. 4393-4407, 2019.

[19] P. Han, Z. Lin, J. Zhang, Y. Xia and L. Wang, "Equivalent Modeling of Photovoltaic Power Plant Based on Factor Analysis and Correlation Clustering," in IEEE Access, vol. 7, pp. 56935-56946, 2019.

[20] P. Chao et al., "Fault Ride-Through Behaviors Correction-based SingleUnit Equivalent Method for Large Photovoltaic Power Plants," in IEEE 
Trans. on Sustainable Energy, 2020.

[21] P. Chao et al., "A comprehensive review on dynamic equivalent modeling of large photovoltaic power plants," Solar Energy, 2020.

[22] Z. Zhu, G. Geng and Q. Jiang, "Power System Dynamic Model Reduction Based on Extended Krylov Subspace Method," in IEEE Trans. on Power Systems, vol. 31, no. 6, pp. 4483-4494, Nov. 2016.

[23] A. M. Stankovic, A. T. Saric and M. Milosevic, "Identification of nonparametric dynamic power system equivalents with artificial neural networks," in IEEE Trans. on Pow. Syst., 18, 4, 1478-1486, Nov. 2003.

[24] G. Chaspierre, P. Panciatici and T. Van Cutsem, "Aggregated Dynamic Equivalent of a Distribution System Hosting Inverter-Based Generators," 2018 Power Syst. Comput. Conf. (PSCC), Dublin, 2018.

[25] U. D. Annakkage et al., "Dynamic System Equivalents: A Survey of Available Techniques," in IEEE Trans. on Power Delivery, vol. 27, no. 1, pp. 411-420, Jan. 2012.

[26] Australian Energy Market Operator (AEMO), "Final report- Queensland and South Australia system separation on 25 August 2018", AEMO Information \& Support Hub, Tech. Rep., Australia, 2019.

[27] C. Li, J. Xu and C. Zhao, "A Coherency-Based Equivalence Method for MMC Inverters Using Virtual Synchronous Generator Control," in IEEE Trans. on. Power Delivery, vol. 31, no. 3, pp. 1369-1378, June 2016.

[28] S. I. Nanou, A. G. Papakonstantinou, S. A. Papathanassiou, "A generic model of two-stage grid-connected PV systems with primary frequency response and inertia emulation", Electric Power Systems Research, vol. 127, pp. 186-196, 2015.

[29] L. Chen, et al., "Design and Implementation of Three-Phase Two-Stage Grid-Connected Module Integrated Converter," in IEEE Transactions on Power Electronics, vol. 29, no. 8, pp. 3881-3892, Aug. 2014.

[30] Online Appendix, available https://www researchgate net/publication/344956112 ONLINE APPEN DIX

[31] L. Chen, et al., "Design and Implementation of Three-Phase Two-Stage Grid-Connected Module Integrated Converter," in IEEE Transactions on Power Electronics, vol. 29, no. 8, pp. 3881-3892, Aug. 2014.

[32] Conn, Andrew R., Katya Scheinberg, and Luis N, Vicente. Introduction to derivative-free optimization. vol. 8. SIAM Journ. on Optimiz., 2009.

[33] K. Price, R. M. Storn, and J. A. Lampinen, "Differential Evolution - A Practical Approach to Global Optimization", Springer, 2005.

[34] G. Chaspierre, P. Panciatici, T.V. Cutsem, "Dynamic equivalent of a distribution grid hosting dispersed photovoltaic units", IREP'17 Symposium, Espinho, Portugal, 2017.

[35] P. Kundur, "Power system stability and control", McGraw-Hill Inc, New York, 1994.

[36] P. Aristidou, D. Fabozzi and T. Van Cutsem, "Dynamic Simulation of Large-Scale Power Systems Using a Parallel Schur-Complement-Based Decomposition Method," in IEEE Trans. on Parallel and Distributed Systems, vol. 25, no. 10, pp. 2561-2570, Oct. 2014.

[37] PowerFactory, "PowerFactory 2020: integrated power system analysis software for transmission, distribution, industry, generation, integration of renewables", 2020.

[38] M. Gibbard and D. Vowles, "Simplified 14-generator model of the South East Australian power system," Adelaide Univ., pp. 1-138, 2014.

[39] M. Ghazavi Dozein, P. Mancarella, "Frequency response capabilities of utility-scale battery energy storage systems, with application to the August 2018 separation event in Australia", $9^{\text {th }}$ international conference on power and energy systems (ICPES2019), Perth, Australia, 2019.

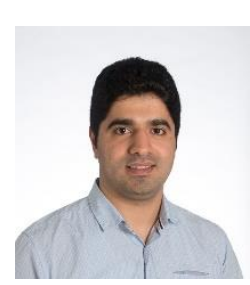

Mehdi Ghazavi Dozein received the M.Sc. degree (2014) in electrical engineering-power systems from the University of Tehran, Iran. He is currently pursuing the Ph.D. degree in power systems at The University of Melbourne, Melbourne, Australia. His research interests include system dynamics and stability in converter-rich grids, power system strength, grid integration of renewables, and modelling and control of inverter-based technologies.

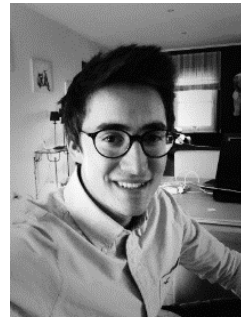

Gilles Chaspierre holds a M.Sc. degree in Electrical Engineering from University of Liège, Belgium. He received his $\mathrm{PhD}$ degree at the same University in 2020 . He is now a postdoctoral researcher at KULeuven/Energyville. His main scientific interests are the study of lowinertia power systems dynamics, the modelling and control of inverter-based generators and the identification of aggregated dynamic equivalents for power system dynamics and stability studies.

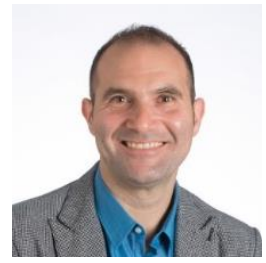

Pierluigi Mancarella (M'08-SM'14) received the M.Sc. (2002) and Ph.D. (2006) degrees in electrical energy systems from the Politecnico di Torino, Turin, Italy. He is currently the Chair Professor of Electrical Power Systems at The University of Melbourne, Melbourne, Australia, and Professor of Smart Energy Systems at The University of Manchester, Manchester, U.K. His research interests include multi-energy systems, grid integration of renewables, energy infrastructure planning under uncertainty, and resilience of low-carbon networks. Dr. Mancarella is an Editor of the IEEE TRANSACTIONS ON POWER SYSTEMS and the IEEE TRANSACTIONS ON SMART GRID, an IEEE Power and Energy Society Distinguished Lecturer, and the Convenor of the Cigre Working Group $\mathrm{C} 6 / \mathrm{C} 2.34$ "Flexibility provision from distributed energy resources".

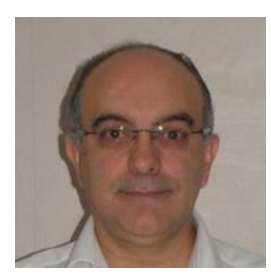

Patrick Panciatici graduated from Ecole Supérieure d'Electricité (Supelec), France. He joined EDF R\&D in 1985, and RTE in 2003, where he is scientific advisor in the R\&D direction. He coordinates and supervises long-term research activities, in particular large projects supported by EU (PEGASE, Twenties, iTesla, e-HIGHWAY2050, Migrate, etc.). He is a member of CIGRE and Fellow member of IEEE and SEE (France). He is the representative of RTE in PSERC (Power Systems Engineering Research Center), USA.

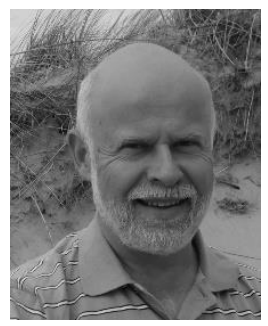

Thierry Van Cutsem graduated in Electrical-Mechanical Engineering from the University of Liège, Belgium, where he obtained the Ph.D. degree and he is now adjunct professor. Since 1980, he has been with the Fund for Scientific Research (FNRS), of which he is now a Research Director. His research interests are in power system dynamics, stability, security, monitoring, control and simulation. He has been active in several CIGRE and IEEE Working Groups. He is a Fellow of the IEEE and SEE (France). 